\title{
Artificial Intelligence for Diagnosis of Inherited Retinal Disease: An Exciting Opportunity and One Step Forward
}

\author{
Tien-En Tan, MBBS (Hons), MMed (Ophth), FRCOphth ${ }^{1,2}$ \\ Hwei Wuen Chan, MBBS, MMed (Ophth), FAMS, FRCOphth ${ }^{3,4}$ \\ Mandeep S. Singh, MD, PhD 5 \\ Tien Yin Wong, MD, PhD ${ }^{1,2}$ \\ Jose S. Pulido, MD, MBA, MPH ${ }^{6}$ \\ Michel Michaelides, MD (Res), FRCOphth, FACS ${ }^{4,7}$ \\ Elliott H. Sohn, $M D^{8,9}$ \\ Daniel Shu Wei Ting, MD ( $1^{\text {st }}$ Hons $), \mathrm{PhD}^{1,2}$
}

1. Singapore Eye Research Institute, Singapore, Singapore National Eye Centre, Singapore

2. Duke-National University of Singapore Medical School, Singapore

3. Department of Ophthalmology, National University of Singapore, Singapore

4. UCL Institute of Ophthalmology, University College London, London, UK

5. Wilmer Eye Institute, Johns Hopkins University School of Medicine, Baltimore, Maryland, USA

6. Wills Eye Hospital, Thomas Jefferson University, Philadelphia, Pennsylvania, USA

7. Moorfields Eye Hospital, London, UK

8. University of lowa Hospitals and Clinics, lowa City, lowa, USA

9. Institute for Vision Research, University of lowa, lowa City, lowa, USA 


\section{Corresponding Author:}

Daniel Shu Wei Ting, MD ( $1^{\text {st }}$ Hons $)$, PhD

Associate Professor, Duke-NUS Medical School, Singapore

Head, Al and Digital Innovation, Singapore Eye Research Institute

Consultant, Surgical Retina, Singapore National Eye Centre

E-mail: daniel.ting45@gmail.com

Address: 11 Third Hospital Avenue, Singapore 168751

Telephone: (+65) 62277255

Word Count: 1,926 words 


\section{INTRODUCTION}

Inherited retinal disease (IRD) affects approximately 1 in 3,000 individuals in North America and Europe, and is a significant cause of visual impairment and blindness among children and working-age adults, with huge personal and societal impact.[1,2] Accurate clinical phenotypic and genotypic diagnosis of IRD is challenging, but increasingly important and relevant. Traditionally, genotypic diagnosis has been considered "nice to have", but not "essential", with implications usually related to patient prognostication and genetic counselling. However, an accurate genetic diagnosis is now of paramount importance because of rapid advances in potential gene replacement and other therapies for these previously untreatable conditions. In 2017, the first gene therapy for IRD was approved by the United States Food and Drug Administration (FDA) for the treatment of RPE65-mediated retinal dystrophy and shortly after by the European Medicines Agency (EMA) as well.[3] Multiple clinical trials are currently underway for other IRDs, including Choroideremia, Stargardt disease, and Retinitis Pigmentosa (RP).[4,5] Besides gene replacement therapy, progress in other areas such as antisense oligonucleotide (AON) therapy and gene editing with clustered regularly interspaced short palindromic repeats (CRISPR) and CRISPR-associated proteins (Cas) also rely on accurate genetic diagnosis.[5,6]

\section{UNMET CLINICAL CHALLENGES}

Successful genotypic diagnosis remains elusive for many patients globally, due in part to remaining gaps in knowledge, but also due to limited access to testing, which 
remains relatively expensive, along with scarcity and an uneven distribution of institutions with expertise in IRD. In the majority of tertiary centres in the Western world, patients have a high chance of an accurate genetic diagnosis. Recent studies have demonstrated the successful characterization of large cohorts of patients with IRD using systematic clinical phenotyping and genetic testing protocols.[7-10] Typically, historical, clinical, electrophysiologic and multi-modal imaging data are used to assign each patient a clinical phenotypic category and to facilitate the selection of a genetic testing strategy. For example, in 2017, Stone et al reported successful identification of disease-causing genotypes in $76 \%$ of 1000 consecutive families with IRD.[10] They used a tiered testing strategy, first relying on focused testing for specific genes, based on the phenotypic category. If needed, they then gradually enlarged the molecular hypothesis in a recursive manner up the classification tree, eventually using whole-exome and whole-genome sequencing, only if required. This tiered testing strategy had greater sensitivity, lower average cost, and a much lower false genotype rate (FGR) than a strategy using wholeexome sequencing for all cases. A phenotype-driven genetic testing strategy may therefore be advantageous, but requires a team of experienced clinicians, well versed in accurate clinical phenotyping, seeing diverse groups of patients with IRD. It also requires major infrastructure such as a certified genetic laboratory and communication between the clinician and testing laboratory. Unfortunately, such expertise and resources are not readily available in most countries except in a select few sub-specialty tertiary referral centres worldwide. Most patients with IRD thus do not have access to an accurate clinical phenotypic and genetic diagnosis - these patients often experience an unacceptably long "diagnostic odyssey". This major 
clinical need presents a unique opportunity for artificial intelligence (Al), and particularly deep learning (DL).

\section{AI AND DL IN MEDICINE}

$\mathrm{Al}$ and $\mathrm{DL}$ techniques have been applied extensively to various technical and medical fields, ranging from medical imaging analysis, to natural language processing and speech.[11] Recently, DL using reinforcement learning has shown promising results in prediction of protein structures, with the potential to greatly increase our understanding of how specific genetic variants cause disease.[12] In ophthalmology, DL techniques have been applied to diagnosis of major ocular diseases such as diabetic retinopathy (DR), age-related macular degeneration and glaucoma from colour fundus photographs, fundus autofluorescence (FAF) images and optical coherence tomography (OCT).[13-17]

\section{AI AND DL FOR INHERITED RETINAL DISEASE}

Currently, the application of $\mathrm{Al}$ and $\mathrm{DL}$ techniques for IRD diagnosis is still at a very nascent stage. Most of these efforts have focused on clinical phenotyping. A few studies have investigated automated classification of IRD versus normal controls or acquired retinal disorders, with $\mathrm{Al}$ and $\mathrm{DL}$ applied to various modalities such as ultrawidefield (UWF) fundus photographs, FAF or OCT images.[18-21] For example, Masumoto et al utilized 373 UWF pseudocolour and FAF images with DL to diagnose RP cases from controls, while Shah et al used individual OCT B-scans at the fovea (749 scans from 93 individuals) with DL to distinguish Stargardt disease 
from controls.[18,21] Both of these studies were limited to binary classifications of single IRD disease cases versus controls. Miere et al developed a DL algorithm that used $389 \mathrm{FAF}$ images to classify patients with 3 different IRDs (RP, Best disease and Stargardt disease) from healthy controls.[20] However, their study was fundamentally limited by the fact that only a minority of this cohort was geneticallyconfirmed.

In this issue of the British Journal of Ophthalmology, Fujinami-Yokokawa et al utilized a Japanese Eye Genetics Consortium dataset of 417 images (fundus photographs and FAF images) from 156 subjects, containing 115 geneticallyconfirmed cases of ABCA4-, EYS- and RP1L1-associated retinal dystrophies, and 41 normal age-matched controls, to train and validate a DL system for automated classification amongst these 4 categories. They report encouraging overall sensitivity/specificity values for fundus photographs and FAF images of $88.3 \% / 97.4 \%$ and $81.8 \% / 95.5 \%$, respectively. They also report area under the receiver operating characteristic curve (AUC) values for fundus photographs and FAF images of 0.708 and 0.703 respectively. The authors conclude that the DL system they have developed could provide accurate, easily accessible diagnosis of these 3 important IRDs in Japan, which may one day help to provide earlier diagnosis, more appropriate referrals, and lower cost of investigation and genetic testing at the general ophthalmologist level. This study represents a step in the right direction for Al in the field of IRD. It tackles the 3 most prevalent genetic causes of IRD in the Japanese population, includes only genetically-confirmed cases, and was developed on a clinically well-characterized IRD cohort. It also uses 2 different and 
commonly used imaging modalities in IRD assessment - colour fundus photographs and FAF imaging.

Nevertheless, there are important limitations to this study, which serve to highlight some of the key challenges in applying AI to IRD diagnosis.

First, it is important to note that this is, by nature, an artificially curated dataset. Typical phenotypes associated with $A B C A 4, E Y S$, and $R P 1 L 1$ disease-causing variants are primarily Stargardt disease, RP and occult macular dystrophy respectively, which are markedly different from each other on colour photography and FAF. However, individually, each of these genotypes can result in different, even overlapping, phenotypes. For example, $A B C A 4$ variants can produce a cone dystrophy/cone-rod dystrophy phenotype which can have similarities with RP especially in advanced stages, while EYS variants can also be associated with a cone-rod dystrophy phenotype, and RP1L1 can also be associated with RP.[22] Furthermore, these challenges may be compounded by ethnic-specific variants and founder effects for specific IRD genes. There is limited information on the phenotypic heterogeneity within each genetic group in this study. Therefore, it is difficult to be certain if the DL system developed is truly predicting the causative gene, or if it is relying largely on notable phenotypic differences to make its classification decisions. An alternative approach with potential advantages may be to group subjects initially by phenotype (e.g. Stargardt disease) with different disease-causing variants, and use Al-DL within this subset to predict the causative variant. This may also make any DL system developed more clinically applicable - in practice, patients first present with a particular phenotype (e.g. Stargardt disease), and it is the clinician's task to 
then solve the genetic basis - a task which one day an Al system may be able to facilitate. Of course, such an analysis would require a much larger sample size, and a very well-characterized cohort of patients, both of which are potential challenges in the field of rare/orphan disease.

Second, this Al system deals with mutations in only 3 genes, in a specific ethnic population. In clinical practice, pathogenic variants in more than 250 genes can cause IRD, with significant ethnic and geographic variation. This Al system in its current form is not yet ready for general deployment on "unknown" IRD patients, that may exhibit significant genotypic and phenotypic heterogeneity.

Third, as with most other DL systems in the early stages of development, this study relies on a selected subset of images of good quality for training and validation. The authors excluded $31.5 \%$ of colour fundus photographs, and $60.4 \%$ of FAF images in their initial quality assessment. Therefore, the encouraging sensitivity and specificity results reported in their validation need to be interpreted in context. The challenge here, and indeed with other DL systems pushing towards clinical translation, will be to prove that the algorithms developed are robust and generalisable, by testing on external datasets with a variety of image quality and acquisition protocols. Ultimately, DL systems employed as "physician assistive tools", as opposed to fully autonomous systems (e.g. for DR screening), are less vulnerable to image quality concerns, but this still needs to be addressed in future studies.

Fourth, the AUC values reported in this study of 0.708 and 0.703 are relatively low as compared to DL systems for other ocular conditions. This may be due to 
limitations of small sample size or reflect an inherent difficulty in the task at hand.

Future studies with larger sample sizes, and appropriate performance benchmarking against human experts will help to resolve this issue.

\section{FUTURE DIRECTIONS FOR AI APPLICATIONS IN IRD}

Evidently, to support further development of Al tools for IRD, there is a need for more well-characterized, sizable datasets. IRDs are individually rare diseases, and there exists also significant geographic variation in relative prevalence patterns of genotypes and phenotypes. Therefore, in order to develop clinically useful and robust $\mathrm{Al}$ algorithms, there is a need for greater international collaboration.

Key areas for future development include:

1. Global standardization of nomenclature, classification systems, and data collection for IRD databases by international IRD consortia/consensus groups this will provide strong ground truth data for Al development

2. Inclusion of additional information from the retinal periphery by greater reliance on UWF colour and FAF images

3. Inclusion of key clinical history data as algorithm input (such as age of onset, family history etc.)

4. Multi-class and multi-modal (including UWF imaging, FAF imaging, OCT, electrophysiology and visual field) Al algorithms for classification of IRD

5. Triaging Al algorithms to stratify the genotype of IRDs to determine suitability for specific gene therapies 
6. Predictive $\mathrm{Al}$ algorithms on IRD progression

7. Testing of Al algorithms on independent external datasets, with a variety of image quality and acquisition protocols

8. Benchmarking of Al algorithms against expert human performance

\section{CONCLUSIONS}

Al systems capable of providing accurate phenotypic or genotypic classification of IRDs based on clinical and multi-modal imaging data could become useful assistive tools for clinicians, particularly in clinics or centres where sub-specialty expertise in IRD is lacking. This would help to fill expertise and resource gaps, and guide general clinicians to order appropriate genetic tests by a targeted testing strategy, which would help to keep testing costs low, minimize the FGR, and potentially, also further improve the rates of genetic diagnosis.

If Al tools can also identify causative sequence variants in certain "difficult" cases and outperform human experts, then analysis of "heatmaps" and reasons for the Al classification decisions may help to identify novel subtle phenotypic abnormalities that point to the underlying genetic diagnosis.

Hopefully, with a concerted effort and greater international collaboration, such effective Al tools for more rapid and accurate diagnosis of IRD can be developed to optimize patient care, with the promise that blindness from IRD can be ultimately prevented or eliminated. 


\section{FUNDING STATEMENT}

This research received no specific grant from any funding agency in the public, commercial or not-for-profit sectors.

\section{COMPETING INTERESTS STATEMENT}

TYW and DSWT are co-inventors, with patents pending, for a deep learning system for diabetic retinopathy, glaucoma, and age-related macular degeneration (SG NonProvisional Application number 10201706186V), and a computer-implemented method for training an image classifier using weakly annotated training data (SG Provisional Patent Application number 10201901083Y), and are co-founders and shareholders of EyRIS, Singapore. All other authors declare no relevant competing interests.

\section{CONTRIBUTORSHIP STATEMENT}

TET, HWC, MSS, TYW, JSP, MM, EHS, DSWT contributed to drafting and revising the manuscript. 


\section{REFERENCES}

1 Moore AT. Genetic Testing for Inherited Retinal Disease. Ophthalmology 2017;124:1254-5. doi:10.1016/j.ophtha.2017.06.018

2 Galvin O, Chi G, Brady L, et al. The Impact of Inherited Retinal Diseases in the Republic of Ireland (ROI) and the United Kingdom (UK) from a Cost-of-Illness Perspective. Clin Ophthalmol 2020;14:707-19. doi:10.2147/OPTH.S241928

3 Russell S, Bennett J, Wellman JA, et al. Efficacy and safety of voretigene neparvovec (AAV2-hRPE65v2) in patients with RPE65-mediated inherited retinal dystrophy: a randomised, controlled, open-label, phase 3 trial. Lancet 2017;390:849-60. doi:10.1016/S0140-6736(17)31868-8

4 MacLaren RE, Groppe M, Barnard AR, et al. Retinal gene therapy in patients with choroideremia: initial findings from a phase 1/2 clinical trial. Lancet 2014;383:1129-37. doi:10.1016/S0140-6736(13)62117-0

5 Hafler BP. CLINICAL PROGRESS IN INHERITED RETINAL DEGENERATIONS: GENE THERAPY CLINICAL TRIALS AND ADVANCES IN GENETIC SEQUENCING. Retina (Philadelphia, Pa) 2017;37:417-23. doi:10.1097/IAE.0000000000001341

6 Cideciyan AV, Jacobson SG, Drack AV, et al. Effect of an intravitreal antisense oligonucleotide on vision in Leber congenital amaurosis due to a photoreceptor cilium defect. Nat Med 2019;25:225-8. doi:10.1038/s41591-018-0295-0

7 Pontikos N, Arno G, Jurkute N, et al. Genetic Basis of Inherited Retinal Disease in a Molecularly Characterized Cohort of More Than 3000 Families from the 
United Kingdom. Ophthalmology Published Online First: 16 April 2020. doi:10.1016/j.ophtha.2020.04.008

8 Weisschuh N, Obermaier CD, Battke F, et al. Genetic architecture of inherited retinal degeneration in Germany: A large cohort study from a single diagnostic center over a 9-year period. Hum Mutat 2020;41:1514-27. doi:10.1002/humu.24064

9 Sharon D, Ben-Yosef T, Goldenberg-Cohen N, et al. A nationwide genetic analysis of inherited retinal diseases in Israel as assessed by the Israeli inherited retinal disease consortium (IIRDC). Hum Mutat 2020;41:140-9. doi:10.1002/humu.23903

10 Stone EM, Andorf JL, Whitmore SS, et al. Clinically Focused Molecular Investigation of 1000 Consecutive Families with Inherited Retinal Disease. Ophthalmology 2017;124:1314-31. doi:10.1016/j.ophtha.2017.04.008

11 LeCun Y, Bengio Y, Hinton G. Deep learning. Nature 2015;521:436-44. doi:10.1038/nature14539

12 Callaway E. 'It will change everything': DeepMind's Al makes gigantic leap in solving protein structures. Nature 2020;588:203-4. doi:10.1038/d41586-02003348-4

13 Gulshan V, Peng L, Coram M, et al. Development and Validation of a Deep Learning Algorithm for Detection of Diabetic Retinopathy in Retinal Fundus Photographs. JAMA 2016;316:2402-10. doi:10.1001/jama.2016.17216 
14 Ting DSW, Cheung CY-L, Lim G, et al. Development and Validation of a Deep Learning System for Diabetic Retinopathy and Related Eye Diseases Using Retinal Images From Multiethnic Populations With Diabetes. JAMA 2017;318:2211-23. doi:10.1001/jama.2017.18152

$15 \mathrm{Li}$ Z, He Y, Keel S, et al. Efficacy of a Deep Learning System for Detecting Glaucomatous Optic Neuropathy Based on Color Fundus Photographs. Ophthalmology 2018;125:1199-206. doi:10.1016/j.ophtha.2018.01.023

16 Keenan TDL, Chen Q, Peng Y, et al. Deep Learning Automated Detection of Reticular Pseudodrusen from Fundus Autofluorescence Images or Color Fundus Photographs in AREDS2. Ophthalmology 2020;127:1674-87. doi:10.1016/j.ophtha.2020.05.036

17 De Fauw J, Ledsam JR, Romera-Paredes B, et al. Clinically applicable deep learning for diagnosis and referral in retinal disease. Nat Med 2018;24:1342-50. doi:10.1038/s41591-018-0107-6

18 Shah M, Roomans Ledo A, Rittscher J. Automated classification of normal and Stargardt disease optical coherence tomography images using deep learning. Acta Ophthalmol 2020;98:e715-21. doi:10.1111/aos.14353

19 Miere A, Capuano V, Kessler A, et al. Deep learning-based classification of retinal atrophy using fundus autofluorescence imaging. Comput Biol Med 2021;130:104198. doi:10.1016/j.compbiomed.2020.104198

20 Miere A, Le Meur T, Bitton K, et al. Deep Learning-Based Classification of Inherited Retinal Diseases Using Fundus Autofluorescence. J Clin Med 2020;9. doi:10.3390/jcm9103303 
21 Masumoto H, Tabuchi H, Nakakura S, et al. Accuracy of a deep convolutional neural network in detection of retinitis pigmentosa on ultrawide-field images. PeerJ 2019;7:e6900. doi:10.7717/peerj.6900

22 Yang L, Fujinami K, Ueno S, et al. Genetic Spectrum of EYS-associated Retinal Disease in a Large Japanese Cohort: Identification of Disease-associated Variants with Relatively High Allele Frequency. Sci Rep 2020;10:5497. doi:10.1038/s41598-020-62119-3 